\title{
Energy Efficiency of Living Creatures from the Mechanical Viewpoint
}

\author{
Sung-Ho Park \\ Department of Railroad Vehicle Engineering, Dongyang University, Youngju-Si, Kyungbook, Korea \\ shpark@dyu.ac.kr
}

Keywords: Living Creatures, Locomotion, Energy, Energy Efficiency, Energy Consumption, Torques, Stroke, Pitch, Duty Factor, Stability, Leg, Moment of Inertia, Link, Walking Machine, Thigh, Shank, Joints, Alligator, Insects, Horse.

\begin{abstract}
Animals or insects can walk or run more elegant and advanced than any other legged mechanical models. They can move on a variety of terrain conditions since they have a distinct ability to adapt to varying conditions by using strictly coordinated leg mechanisms. Nature has an astonishing capability. They continuously have modified their body structures and living patterns, which makes it possible to survive and still continue their developments. Structural and locomotive characteristics of four-legged animals are copied and modelled from the mechanical view point, as body and links. The model is the robot, having 4-legs. Under the given structure and the locomotion conditions, the total energy to complete one cycle is calculated and finally suggested the optimal locomotive conditions.
\end{abstract}

\section{Introduction}

Living creatures learn themselves the way how to fly, live, make surprising substances required to live a life. Inspiration from nature, where the greatest effects are created with least effort, continues to fascinate [1]. Bio-inspiration is the major concern in engineering and especially in Robotics. Applying engineering experience to the world of animals has shown, how well adapted and made animals are for the roles they play [2]. But the practical application to engineering remains unrealized.

For the survival of living creatures, quick decisions are essential and efficient control systems to carry them are also required, which are done by their brains. There exists big difference between man-made machine and living creatures. Robots repeat any given work over and over again. If other work is required, control program must be modified and applied to the system, which takes a long time. But animals react abruptly and execute many diverse works according to surrounding circumstance. Their behavior is more adaptive.

Newcastle [3] first started horse gait study and a series of researches on animal locomotion have been done. Sukhanov [4] introduced over 100 gait patterns with detailed figures and gait diagrams, which connect well with previous observations by biologists and robot researchers. Using thin foil 
shape memory alloy actuators and Inspiration from biology, the feasibility of insect-scale ambulatory micro-robot was suggested [5]. Also, bio-inspired caterpillar robot [6] and cockroach inspired robot [7] were introduced. Boston Dynamics made the four-legged robot Littledog [8] and BigDog [9]. Several models were made, but their functions were very limited.

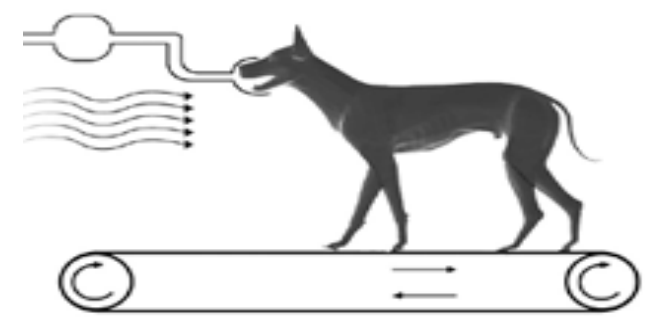

Figure 1: Biological method to estimate energy consumption.

As locomotion speed is going up, animals spend more energy. The rate of oxygen consumption over a wide range of walking and trotting speeds, as horses walk or run on the treadmill, was measured by Griffin [10], as shown in Fig.1. Horses wear a facemask and walk on the treadmill, facing an air, flowing to it. Several researches about the relationships among gait patterns, gait transitions, speeds and energy consumption were done in experimental biology. Also, they found many principles of animal locomotive characteristics, from the biological viewpoint. Animal is basically a serial linkage of rigid bodies connected by revolute joints. Dynamic principles are applied to the model and simulated. Finally, optimal parameters are suggested.

\section{Major Locomotive Parameters}

Body has a fixed dimension. Other parameters characterizing locomotion are variables, which include duty factor, gait pattern, strokes and direction angle. Followings are the major parameters related to determine the consumed energy during locomotion.

Stroke: During locomotion, front pair legs step on the limited forward distance and the rear pair legs also do. But each pair of legs has different stroke distances, which could be a function of velocity, duty factor or other parameters. There exist four different stroke distances. As in Figure 2, $R_{f f}$ and $R_{f r}$ are the front forward and the front rear stroke distances, respectively. Front and rear pairs of legs have different segment lengths and mass distribution. Rear pair legs are stronger than front pair legs

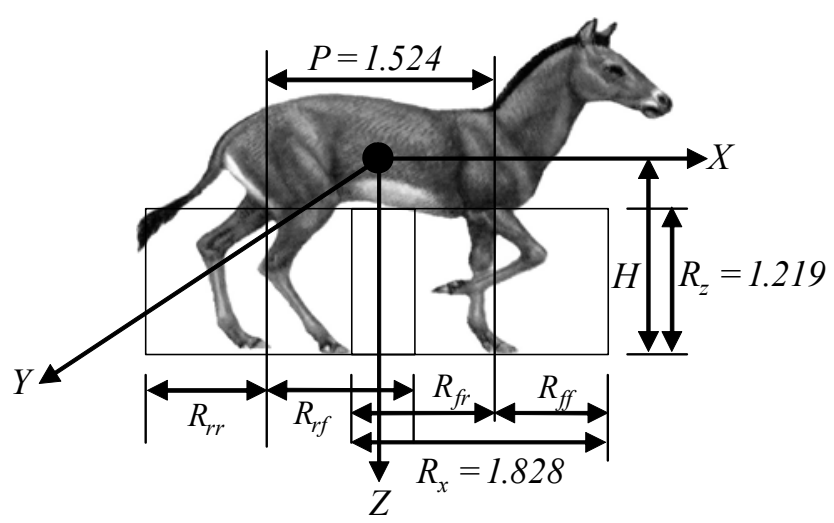

Figure 2: Direction angle of legs. 
Direction angle: The majority of extrinsic muscles move the coxa, whereas the paired intrinsic muscles move leg segments in relation to each other. At slow walking gait, direction of motion coincides with the leg swing, but as the locomotion speed is increased, angle between leg swing and vertical line is also increased, which is the direction angle. There exists optimal direction angle which makes the energy efficiency maximum.

Gait: Gait is a complex, strictly coordinated rhythmic movement of the entire body of the animal treated as an integral complex of reflex acts that occur in accordance with the conditions of the environment and which are capable of producing progressive movements of different type inherent in each species [11]. Animals use their own specific walking patterns, which have been developed by experimental biologists. Due to the control problems caused by a series of muscle bundles in animal, it is extremely difficult to the same systems to the suggested mechanical model. Simplified four-legged bio-inspired mechanical model, as shown in Figure 2, is simulated and suggested the optimal conditions, from the principle of the minimum energy expenditure

\section{Dynamics of Model}

Mechanical properties of the right side leg are the same as those of left side leg. In the support phase, legs move backward from the forward stroke limit to the backward stroke limit with a constant velocity. But in the transfer phase, the legs are lifted at the backward stroke limit and move according to the desired foot trajectory of polynomial time functions.

Legs are accelerated and decelerated during transfer phase, with acceleration, deceleration, or constant velocity, all contributing to the dynamic leg system. Kinematics and dynamics of a serial link manipulator are applied to the leg, which gives the forces/torques acting at the joints. Dynamic model is usually a set of nonlinear differential equation called the equation of motion.

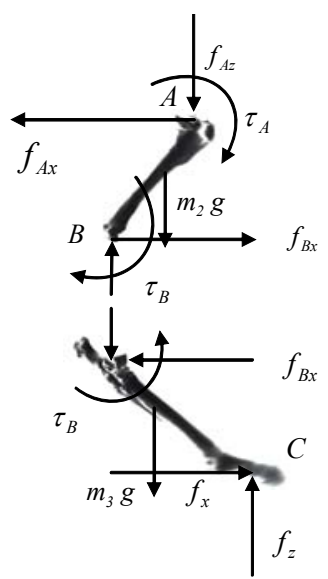

Figure 3: Free-Body Diagram for the Thigh and Shank.

D'Alembert's principle is applied to analyze three dimensional motions. Figure 3 shows the freebody diagram of legs, which includes thigh and thank. The equation of motion and the constraints for the locomotion can be derived. For the shank $B C$, three equations of D'Alembert's principle on the $x-z$ plane are

$$
\begin{aligned}
& \sum F_{x}=\sum\left(F_{x}\right)_{e f f} \\
& \sum F_{z}=\sum\left(F_{z}\right)_{e f f} \\
& \sum M_{y}=\sum\left(M_{y}\right)_{e f f}
\end{aligned}
$$


By applying the equation (1)-(3) to the free-body-diagram of link $B C$, forces and torques acting at the joint are derived, which can be converted to the consumed energy and finally specific resistance.

$$
\begin{gathered}
f_{B x}=f_{x}+m_{3}\left(l_{2} C_{2} \dot{\theta}_{2}^{2}+l_{2} S_{2} \ddot{\theta}_{2}+l_{c 3} S_{23} \ddot{\theta}_{23}+l_{c 3} C_{23} \dot{\theta}_{23}^{2}\right) \\
f_{B z}=f_{z}-m_{3}\left(l_{2} S_{2} \dot{\theta}_{2}^{2}-l_{2} C_{2} \ddot{\theta}_{2}+l_{c 3} C_{23} \ddot{\theta}_{23}+l_{c 3} S_{23} \dot{\theta}_{23}^{2}\right)-m_{3} g \\
\tau_{B}=\left\{I_{y 3}+m_{3}\left(l_{2} C_{3}+l_{c 3}\right) l_{c 3}\right\} \ddot{\theta}_{2}+\left(I_{y 3}+m_{3} l_{c 3}^{2}\right) \ddot{\theta}_{23}+m_{3} l_{2} l_{c 3} S_{3} \dot{\theta}_{2}^{2}+m_{3} g l_{c 3} C_{23}+f_{x} l_{3} S_{23}+f_{z} l_{3} C_{23}
\end{gathered}
$$

In the lifting of transfer phase, foot is accelerated and the velocity in x-direction changes from negative to positive with respect to the body. In the placing of transfer phase, the velocity in $\mathrm{x}-$ direction changes sign from positive to negative and the velocity in y-direction is zero at both ends. There are six boundary conditions at both end positions. Fifth degree polynomial equation of time is used to derive the foot trajectory.

$$
x(t)=\frac{6}{T_{2}{ }^{5}}\left(V T_{2}+R\right) t^{5}-\frac{15}{T_{2}{ }^{4}}\left(V T_{2}+R\right) t^{4}+\frac{10}{T_{2}{ }^{3}}\left(V T_{2}+R\right) t^{3}-V t+R_{f}-R
$$

The cycle time of the support phase is $T_{1}$ and $T_{2}$ is the cycle time of transfer phase. Other coordinates for the specific range can be derived by using related boundary and initial conditions.

\subsection{Energy Consumption}

Lagrange-Euler method, Recursive Lagrange method, Newton-Euler method and Generalized D'Alembert's method can be applied to the dynamic equations of motion. Silver [12] showed that, the form of Lagrange equations can be equivalent with the Newton-Euler equation. Due to the recursive nature of the equations and the method of systematically computing the force/torque, computation becomes much simpler with the Iterative Newton-Euler method.

We can rearrange the force and torque equations so that they can appear as iterative relationship from higher-numbered neighbor to lower-numbered neighbor. These equations are evaluated link by link starting from link $n$ and working inward toward the base, which is the body of the legged walking machine.

$$
\begin{gathered}
f_{i}^{i}=F_{i}{ }^{i}+R_{i+1}{ }^{i} f_{i+1}{ }^{i+1} \\
n_{i}{ }^{i}=N_{i}{ }^{i}+R_{i+1}{ }^{i} n_{i+1}{ }^{i+1}+p_{c i}{ }^{i} \times F_{i}^{i}+p_{i+1}{ }^{i} \times R_{i+1}{ }^{i} \times f_{i+1}{ }^{i+1}
\end{gathered}
$$

Finally, the $z$ component of $n_{i}{ }^{i}$ is the required joint torque.

$$
\tau_{i}=n_{i}^{i T} \vec{z}_{i}^{i}
$$

Also, the tedious calculating procedures give the nonlinear differential equation of motions, which will be applied to calculate the mechanical energy and then torque acting on the joints. Legged locomotion involves large changes in kinematic energy and potential energy. Leg motion requires energy input to accelerate the leg at the beginning of a stroke and energy removal to decelerate the leg at the end of stroke, which converts kinetic energy to heat and causes energy loss. 
Horse runs faster than lizard, but horse weights far more than lizard and spends more energy. It is not so simple to determine the energy efficiency of living creatures. Mechanical energy efficiency of different locomotion systems can be evaluated and compared with by non-dimensional parameters $\varepsilon$, called specific resistance. When $E$ is the total energy required to completing one locomotion cycle, $M$ weight of locomotion system and $L$ distance of locomotion, specific resistance can be written as follows:

$$
\varepsilon=\frac{E}{M L}
$$

\section{Simulation Results}

Figure 4 shows the case of $\beta=0.75$, which guarantees the maximum stability of quadruped. SR $\varepsilon$ of type- 1 has the minimum value of 0.145 at $\theta_{f}=100 \sim 102^{\circ}$. SR $\varepsilon$ of type- 2 has the minimum value of 0.134 at $\theta_{f}=97 \sim 105^{\circ}$. SR $\varepsilon$ of type- 3 has the minimum value of 0.128 at $\theta_{f}=98 \sim 104^{\circ}$. All three types have the average minimum $\operatorname{SR} \varepsilon$ at $\theta_{f}=101^{\circ}$, which guarantees the maximum energy efficiency.

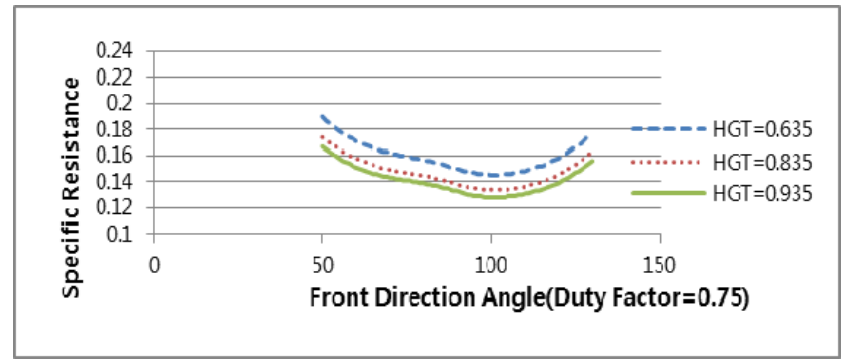

Figure 4: $\theta_{f}-\varepsilon\left(\beta=0.75, R D A=90^{\circ}\right)$ for three different model.

Also, when $\beta$ is increased from 0.75 to 0.8 , SR $\varepsilon$ of type- 1 occurs the minimum value of 0.153 at $\theta_{f}=98 \sim 104^{\circ}$. SR $\varepsilon$ of type- 2 has the minimum value of 0.14 at $\theta_{f}=98 \sim 103^{\circ}$. SR $\varepsilon$ of type- 3 has the minimum value of 0.134 at $\theta_{f}=97 \sim 104^{\circ}$. For the increased duty factor $\beta=0.8$, all three types of model show lower energy efficiency than the cases of $\beta=0.75$. From the simulation results, it is expected that the increase of support phase by 0.05 leads to the $4.6 \%$ decrease of energy efficiency. Therefore, minimum time of support phase to keep the stability will guarantee the maximum energy efficiency.

\section{Conclusion}

Animals are faithful to the principle of the minimum energy expenditure. Simulation program has been developed and applied by using the structure and the locomotive characteristics of living creatures, which can be applied to the legged walking robot. 


\section{Acknowledgements}

The research was supported by the National Research Foundation of Korea(NRF) grant funded by the Korea government(MEST)(No. 2016901202) and the Dongyang University. The authors wish thanks to all the members concerned.

\section{References}

[1] Liyanage, A. L. (2008) Biomimicry as a metaphor for perfect integration in sustainability, LAMBERT Acamemic Press, pp. 1.

[2] Denny, M.and McFadzean A. (2011) Engineering Animals, Harvard Univ. Press, pp. 2-4.

[3] Newcastle, P. G. (1957) La method et l'evention nouvelle de dresser les chevaux, Anvers., cited by Muybridge.

[4] Sukhanov, V. B. (1968) "General System of Symmetrical Locomotion of Terrestrial Vertebrates and Some Features of Movement of Lower Tetrapods", Academy of Sciences, USSR, pp. 10-11, 61-2.

[5] Baisch, A. T. and Wood, J. J. (2011) Design and Fabrication of the Harvard Ambulatory Micro-Robot, Robotics Research, The $14^{\text {th }}$ Int. Sym. ISRR, pp. 715-730.

[6] Houxiang, Z., Wei, W., Juan, G. G. and Jianwei, Z. (2010) A Bio-Inspired Small-Sized Wall Climbing Caterpillar Robot, Mechatronic Systems Applications.

[7] Kingsley, D. A., (2005) A Cockroach Inspired Robot with Artificial Muscles, Ph.D Dissertation, Case Western Reserve University, Ohio, Jan..

[8] Pongas, D., Mistry, M., Schaal, S. (2007) A Robust Quadruped Walking Gait for Traversing Rough Terrain, Proceeding of 2007 IEEE Int'l Conf. on Robotics and Automation, April, pp. 1474-1479.

[9] Raibert, M., Blankespoor, K., Nelson, G, Playter, R. et al. (2008) BigDog, the Rough-Terrain Quadruped Robot, Proceedings of the $17^{\text {th }}$ World Congress The Int'l Fed. of Automatic Control Seoul, Korea, July6-11, pp. 10822-10825.

[10] Giffin, T. M., Kram, R., Wicker, S. J. and Hoyt, D. F. (2004) "Biomechanical and energetic determinants of the walk-trot transition in horses", J. of Exp. Biology, Vol. 207, pp. 4125-4223.

[11] Alexander, R. M. and Jayer, A. S. (1980)“Estimates of Energy Cost for a Quadrupedal Running Gaits”, J. of Zoology, pp. 153-170.

[12] Silver, W. M. (1982) "On the Equivalence of the Lagrangian and Newton-Euler dynamics for manipulators", Int'1 J. of Robotics Research, Vol. 1(2), 60-70. 